\title{
Ultra high energy cosmic ray detector KLYPVE on board the Russian Segment of the ISS
}

\author{
M. I. Panasyuk ${ }^{*},{ }^{a b}$ P. Picozza, ${ }^{c}$ M. Casolino, ${ }^{d e}$ T. Ebisuzaki, ${ }^{d}$ P. Gorodetzky, ${ }^{f}$ \\ B. A. Khrenov, ${ }^{a}$ P. A. Klimov, ${ }^{a}$ S. A. Sharakin ${ }^{a}$ and M. Yu. Zotov ${ }^{a}$ for the JEM-EUSO \\ Collaboration \\ ${ }^{a}$ D.V. Skobeltsyn Institute of Nuclear Physics, M.V. Lomonosov Moscow State University, Moscow \\ 119991, Russia \\ ${ }^{b}$ Faculty of Physics, M.V. Lomonosov Moscow State University, Moscow 119991, Russia \\ ${ }^{c}$ University of Rome Tor Vergata, Italy \\ ${ }^{d}$ RIKEN, 2-1 Hirosawa, Wako, 351-0198 Japan \\ ${ }^{e}$ Istituto Nazionale di Fisica Nucleare - Sezione di Roma Tor Vergata, Italy \\ ${ }^{f}$ APC, Univ Paris Diderot, CNRS/IN2P3, CEA/Irfu, Obs de Paris, Sorbonne Paris Cité, France \\ E-mail: panasyukesinp.msu.ru, piergiorgio.picozza@gmail.com, \\ casolino.marcolgmail.com, ebisuepostman.riken.jp, \\ philippe.gorodetzky@cern.ch, bkhrenoveyandex.ru, \\ pavel.klimov@gmail.com, sharakin@mail.ru, zotov@eas.sinp.msu.ru
}

\begin{abstract}
Modified KLYPVE is a novel fluorescence detector of ultra high energy cosmic rays (UHECRs, energies $\gtrsim 50 \mathrm{EeV}$ ) to be installed on the Russian Segment of the International Space Station. The main goal of the experiment is to register arrival directions and energies of UHECRs but it will be able to register other transient events in the atmosphere as well. The main component of KLYPVE is a segmented two component optical system with a large entrance pupil and a wide field of view, which provides annual exposure approximately twice that of the Pierre Auger Observatory. The project is actively developed by a working group of the JEM-EUSO Collaboration led by Skobeltsyn Institute of Nuclear Physics at Moscow State University (Russia). The current status of KLYPVE with a focus on its scientific tasks, technical parameters and instruments is presented.
\end{abstract}

The 34th International Cosmic Ray Conference,

30 July-6 August, 2015

The Hague, The Netherlands

\footnotetext{
*Speaker.
} 


\section{Introduction}

It was more than 50 years ago that a cosmic ray with an energy of about $100 \mathrm{EeV}$ was first registered at the MIT Volcano Ranch station [1] but we still do not know what are ultra-high energy cosmic rays (UHECRs) and where do they come from. ${ }^{1}$ One of the main difficulties of the puzzle is due to the extremely low flux of UHECRs. Suffices to say that the biggest of the ever existing experiments, the Pierre Auger Observatory, which covers an area of about $3000 \mathrm{~km}^{2}$, registered only 231 event with energies above $52 \mathrm{EeV}$ in more than nine years of operation [4]. Since one can do nothing with the cosmic ray flux, the only way to increase statistics in a reasonable amount of time is to perform an experiment with a bigger exposure.

Another difficulty is purely technical arising from the fact that all ground-based experiments have an incomplete and non-uniform coverage of the celestial sphere. This significantly complicates studies of anisotropy of UHECR arrival directions, which play one of the key roles in the search for their sources.

A possible solution to both problems was put forward by Benson and Linsley, who suggested to observe UHECRs with an orbital instrument capable to register near-ultraviolet fluorescence light [5]. They suggested to look earth-ward from a satellite flying on a circular orbit at a height of $500-600 \mathrm{~km}$ and equipped with a huge mirror (36 m in diameter) with $10^{\prime}$ resolution and $\sim 5000$ photomultiplier tubes $5 \mathrm{~cm}$ in diameter located at the focal surface of the mirror. They estimated that the instrument will have a circular field of view about $100 \mathrm{~km}$ in diameter, a duty cycle of the order of $20-30 \%$, and the energy threshold below $10 \mathrm{EeV}$.

Though very promising, an experiment like this poses a whole number of challenging problems both in technology and science since one has to register a faint flux of photons emitted in the continuously varying conditions of the Earth atmosphere and background radiation. The instrument built with strong demands on mass, energy consumption and other parameters must be able to work in a harsh space environment for a few years.

Since the pioneering work by Benson and Linsley, different aspects of registering UHECRs from space have been studied in much details, see, e.g., [6, 7] and a whole number of projects have been suggested, among them AIR WATCH [8], OWL [9, 10], EUSO and later JEM-EUSO [11]

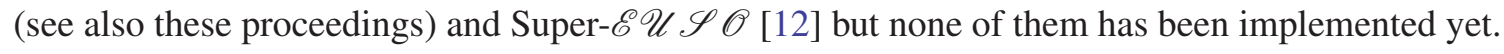
The only fully implemented orbital detector of UHECRs is TUS [13, 14], which was developed in Skobeltsyn Institute of Nuclear Physics of Moscow State University (SINP MSU) in cooperation with several Russian and foreign institutions following the original reflector type telescope scheme suggested by Benson and Linsley.

Being scheduled for launching in late 2015 from the Vostochny Cosmodrome, TUS is not expected to make a breakthrough in UHECR physics because of its pretty modest technical parameters [15] but will serve as a pathfinder for the new class of UHECR experiments. By this reason, a development of the next-step detector was begun at SINP MSU in order to considerably increase the field of view (FOV) of the detector in comparison with TUS and to decrease the energy thresh-

\footnotetext{
${ }^{1}$ In what follows, by UHECRs we mean cosmic rays with energies at and above the Greisen-Zatsepin-Kuz'min (GZK) cut-off at $\sim 50 \mathrm{EeV}[2,3]$. They are sometimes called extreme energy cosmic rays.
} 
old. The new detector called KLYPVE ${ }^{2}$ will have a much bigger and more complicated optical system and will be located on the outer side of the Russian segment of the ISS, see Fig. 1. The instrument consists of an optical system that includes a large mirror and a correcting lens, a focal surface made of photomultiplier tubes (PMTs), electronics including a data acquisition system, an atmosphere monitoring system, a supporting frame and a few other elements.

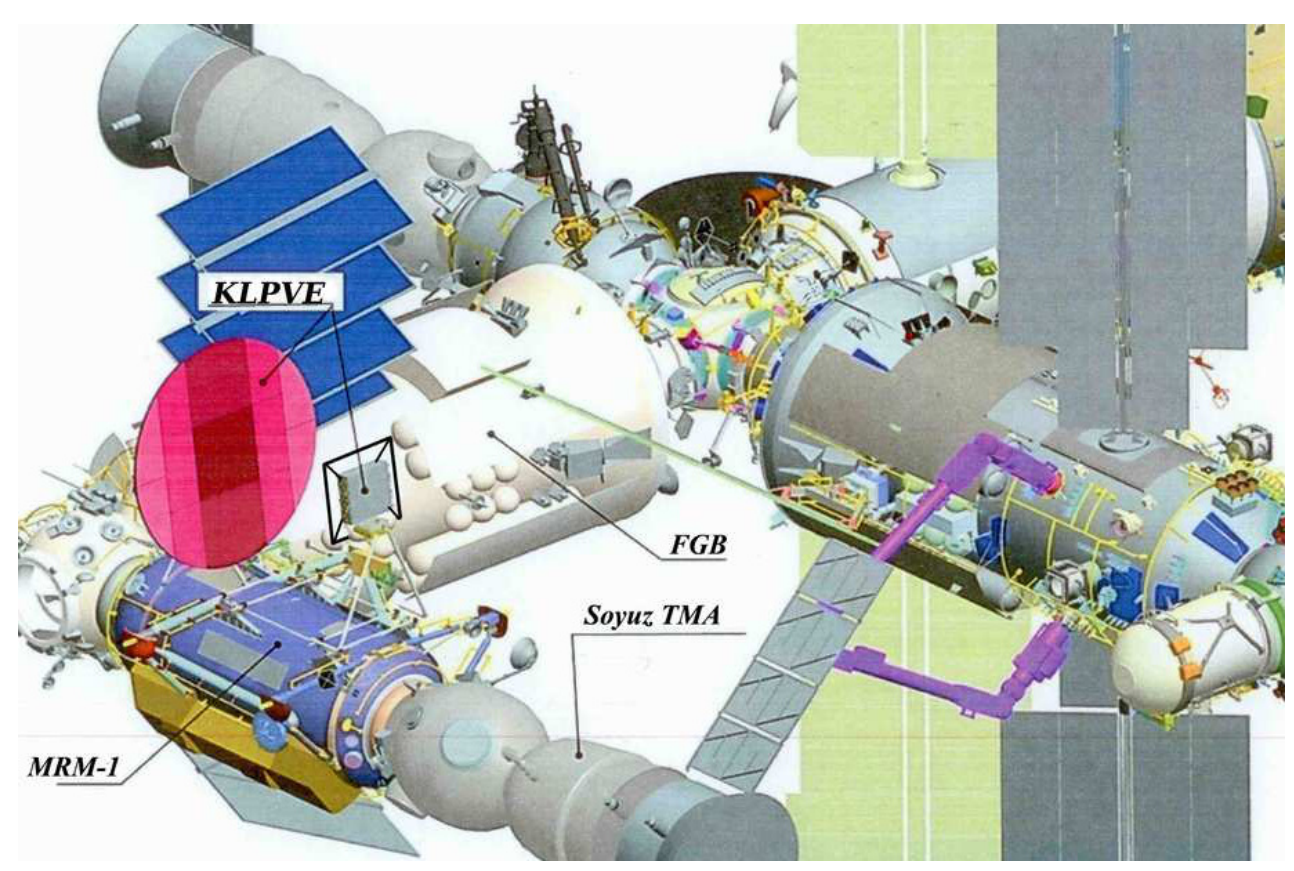

Figure 1: Possible deployment of the KLYPVE detector on the Mini-Research Module 1 (MRM-1) of the ISS.

In 2012, the preliminary design stage of the project was completed, and below we report on the current status of its development.

\section{Scientific Tasks}

The main physics goal of the project is to make a major step in physics of UHECRs by registering a few hundred events coming from the whole celestial sphere.

Similar to JEM-EUSO [16], scientific tasks of the KLYPVE experiment are much broader and include registering UHE photons and neutrinos, relativistic dust granules, hypothetical nuclearites, small meteoroids and other transient events of fluorescence in the atmosphere. Advantages of such orbital detectors depend on the size of the entrance pupil of the optical system and space resolution of optics and the photo receiver in the detector FOV. Several types of both elements of the detector were tested in calculations for various tasks of the KLYPVE experiment and they are compared below.

\footnotetext{
${ }^{2}$ Sometimes written as KLPVE, which is a Russian acronym for "Kosmicheskie Luchi Predel'no Vysokikh Energii”, i.e., cosmic rays of the highest energies.
} 


\section{Optical System}

The main optical part of the initial version of the KLYPVE detector was a mirror-concentrator with a reflective surface of about $10 \mathrm{~m}^{2}$ with the field of view (FOV) $\pm 7.5^{\circ}$, focal distance $3 \mathrm{~m}$ and the angular resolution $5 \mathrm{mrad}$ (pixel size $15 \mathrm{~mm} \times 15 \mathrm{~mm}$, i.e., the same as in the TUS detector). However, it became clear during the preliminary design phase that such characteristics of the instrument do not allow one to solve scientific tasks related to studies of anisotropy of UHECRs because of the comparatively small FOV, low angular resolution and the spot size growing very rapidly with increasing field angle due to the off-axis aberration of a fast optical system. Thus the development of a new optical system for KLYPVE was initiated in order to improve its parameters and the overall performance of the instrument. This work began in late 2013, in a close interaction with members of the JEM-EUSO Collaboration.

Since then, a number of different optical systems have been considered for KLYPVE. All of them have an additional corrective element aimed to eliminate the off-axis aberration, namely a thin $(\sim 1 \mathrm{~cm})$ Fresnel lens made of a material transparent to UV radiation, probably PMMA-000 by Mitsubishi Rayon Co., Ltd. After a series of simulations, two optical systems were selected for the further development. They are described below.

\subsection{Baseline System}

In the so called Baseline system, the diameter of the reflector and the correcting lens are equal to $3.4 \mathrm{~m}$ and $1.7 \mathrm{~m}$ respectively. The total length of the system (more precisely, the axial distance from the pole to the center of the focal surface) is equal to $4 \mathrm{~m}$, the distance from the lens to the focal surface equals $70 \mathrm{~cm}$, see figure 2 . In this case, it is possible to expand the FOV up to $\pm 14^{\circ}$, and the diameter of the image is not larger than $6 \mathrm{~mm}$ in the entire FOV. The angular resolution of the system is $\approx 0.057^{\circ}$, which is equivalent to $\sim 0.4 \mathrm{~km}$ at ground.

A disadvantage of this approach is the difficulty of manufacturing of optical elements, especially lenses. To correct aberrations, both lens surfaces should have a small curvature, wherein the front side is a Fresnel spherical surface (with a radial structure of grooves of $1 \mathrm{~mm}$ depth), and the back side is a spherical diffraction surface (with grooves depth $700 \mathrm{~nm}$ ).

Delivering such a huge system to the ISS is also a complicated task. In case Progress-TM is used, cargo must be placed inside the ISS first. To achieve this, pieces of the instrument have to pass through a cylindrical lock of $70 \mathrm{~cm}$ diameter and $120 \mathrm{~cm}$ length. The currently offered solutions require segmentation of all the major components of the system, including the lens, the mirror and the photodetector, and subsequent deployment in space.

\subsection{Multi-Eye Telescope System}

An interesting option in the design of a complex reflector-type detector like KLYPVE is to divide a wide FOV of one huge telescope into several FOVs of identical smaller telescopes, which would be easier to manufacture and deploy. Such a system consisting of three telescopes was called METS (Multi-Eye Telescope System). METS has several advantages in comparison with the Baseline system: (i) it allows correcting aberrations in narrower FOVs without use of complex surfaces (diffraction, curved Fresnel), (ii) dimensions of the elements can be chosen basing on the 


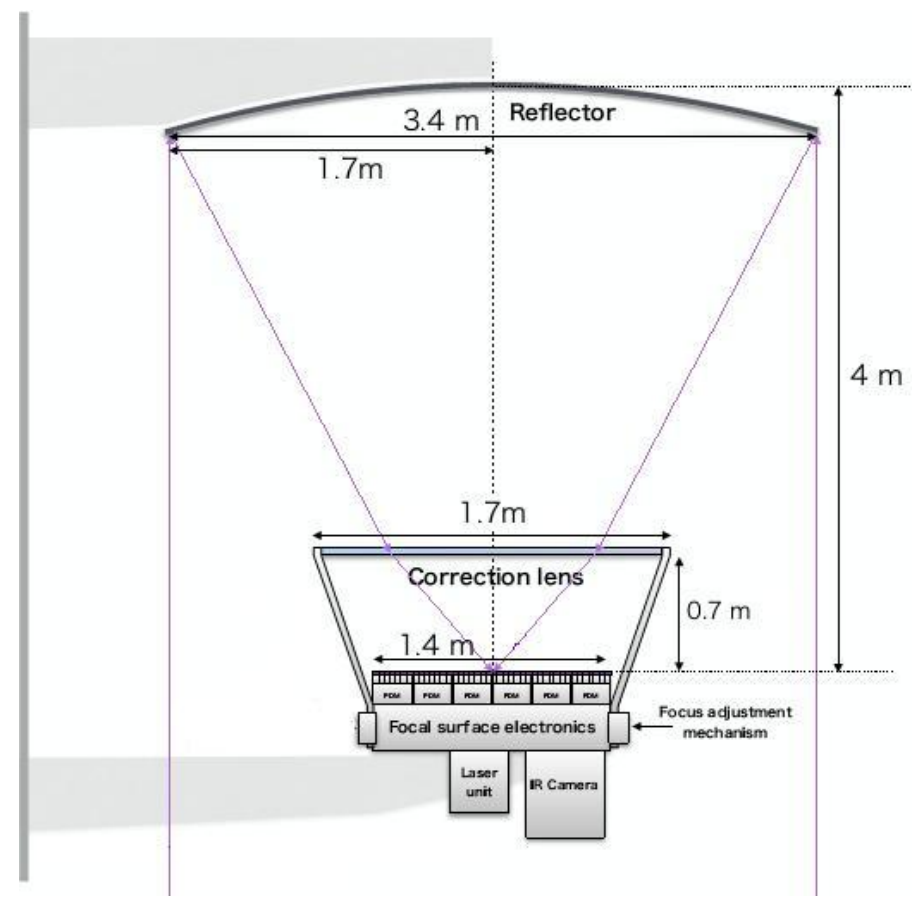

Figure 2: Optics of the Baseline system.

possibility of transporting individual telescopes already assembled on earth. One of drawbacks of METS is the necessity of a trigger working for all three telescopes simultaneously.

In the optimization calculations, the following dimensions of an individual telescope were obtained: $2.4 \mathrm{~m}$ diameter mirror, $1.2 \mathrm{~m}$ lens, $0.9 \mathrm{~m}$ photodetector, the total axial length $3 \mathrm{~m}$. Correction of aberrations in the FOV $\pm 10^{\circ}$ (which corresponds to the diameter of the overall FOV of three telescopes $\sim 35^{\circ}$ ) can be achieved using plane Fresnel surfaces. The angular resolution of METS is $\approx 0.075^{\circ}$, which is equivalent to $\sim 0.5 \mathrm{~km}$ at ground.

An important advantage of METS is the possibility to employ the instrument in different modes of operation provided by a special support system. In the basic mode, shown in the top panel of Fig. 3, the FOVs of individual telescopes are adjacent (or slightly overlapping for crosscalibration). In the coincidence mode (overlapping FOVs), one can significantly reduce the energy threshold. Finally, it is possible to increase the exposure, and hence to collect larger statistics of UHECRs (if a shower trigger can be made) in the tilted mode, in which FOVs of two or all telescopes are inclined relative to the direction at the nadir, see the bottom panel of Fig. 3.

More details about the optical system of KLYPVE can be found in [17].

\section{Focal Surface}

The focal surface (FS) of the updated KLYPVE detector utilizes a design similar to that proposed for the JEM-EUSO detector [18]. It will be produced of almost 120 thousand Hamamatsu R11265-103-M64 multi-anode photomultiplier tubes (MAPMTs), grouped into photodetector modules (PDMs). The number of pixels in one MAPMT is 64, the number of MAPMTs in one 

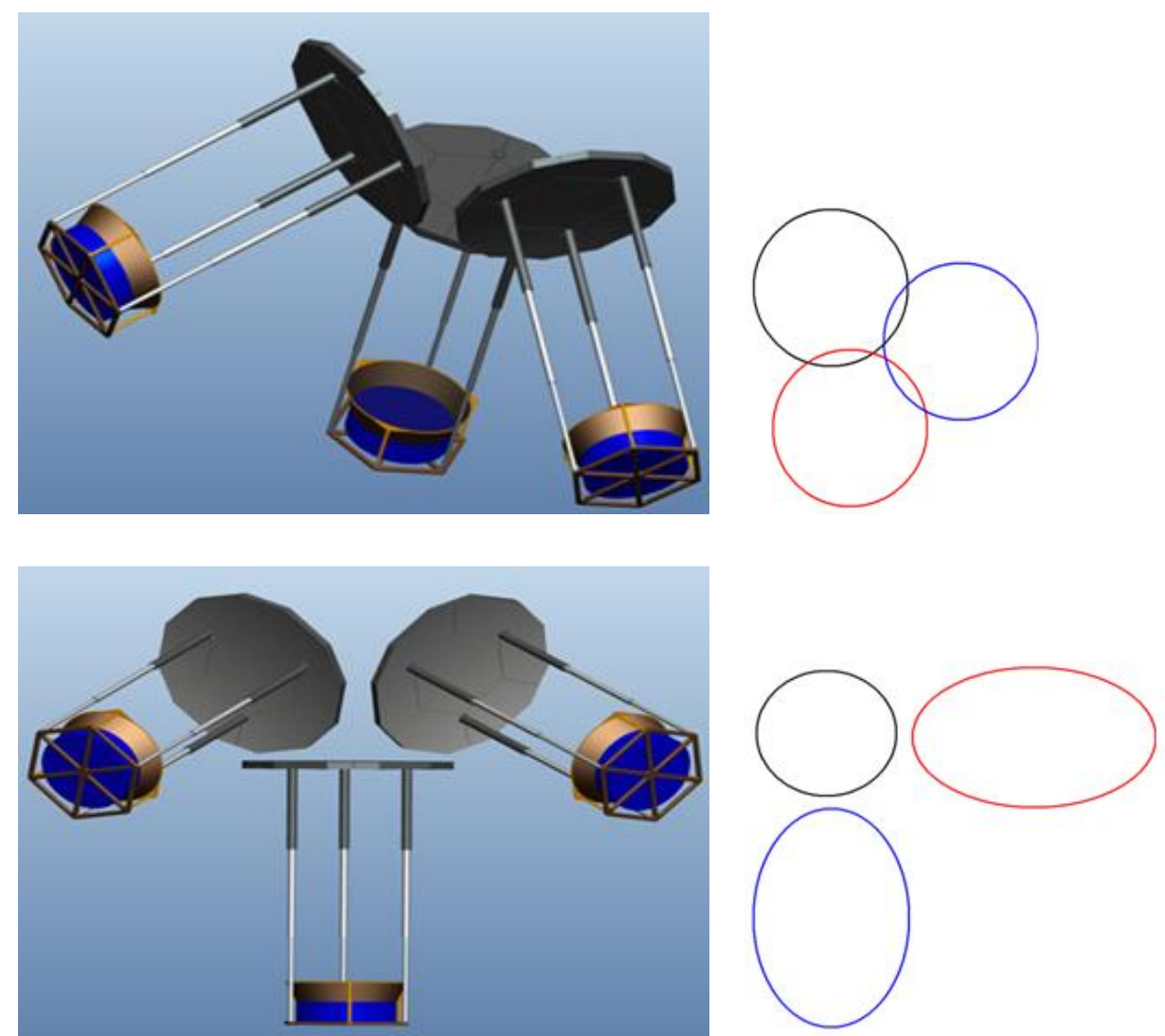

Figure 3: Top: the basic mode of METS operation. The angles of inclination of individual telescopes are exaggerated for clarity. Bottom: The tilted mode of METS operation. The right panels show mutual position of FOVs of separate telescopes.

PDM is 36. The size of a pixel is adjusted to the path length of an extensive air shower during one time sample (one gate time unit, $2.5 \mu \mathrm{s}$ ).

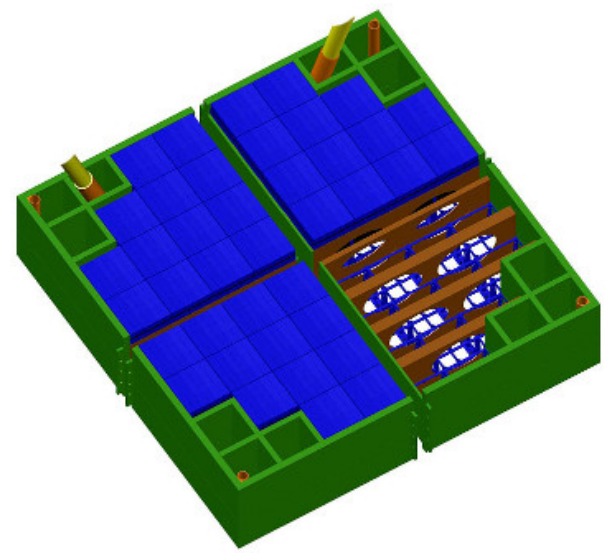

Figure 4: Focal surface of the Baseline KLYPVE. Blue boxes are PDMs, green and brown elements show the supporting structure.

The FS for the Baseline version of KLYPVE is shown in Fig. 4. It consists of 52 PDMs and 
has a slightly concave shape. The size of the photodetector box is approximately $1.4 \mathrm{~m} \times 1.4 \mathrm{~m}$. All four corners of the FS are free from PDMs and will be used for mechanical structures of the supporting frame and other systems of the detector (calibration, adjustment etc).

In the METS version of KLYPVE, the number of PDMs in one detector equals 19, and their total number is 57 .

\section{Atmosphere Monitoring System}

An accurate reconstruction of parameters of UHECRs needs knowledge of the atmosphere conditions in the FOV of the detector. To achieve this, one needs to employ a LIDAR and an atmosphere monitoring system. Both systems have already been developed for JEM-EUSO [19]. A preliminary analysis reveals that they perfectly suit the needs of KLYPVE and can be deployed on the back surface of the focal plane, see Fig. 2.

\section{Conclusions}

The KLYPVE project of an orbital detector of UHECRs finished the stage of the preliminary design and is included in the Russian Federal Space Program. It has entered the stage of the design project in its modified version aimed to improve the technical parameters of the instrument. There is a realistic possibility for KLYPVE to be installed on board the Russian Segment of the ISS in the next 6-7 years. The above improvements of the original KLYPVE detector will allow building an orbital detector with the annual exposure approximately twice that of the Pierre Auger Observatory [15]. Together with an almost uniform exposure of both hemispheres, this gives an additional advantage for anisotropy studies of UHECR arrival directions and it will make an automatic self-calibration between Auger and Telescope Array experiments. In case of its successful implementation, KLYPVE will provide a possibility for a breakthrough in solving the long-standing puzzle of the nature and origin of the most energetic particles ever detected on Earth, will bring new information of some fundamental issues of particle physics and cosmology, collect valuable data about transient events in the atmosphere.

\section{Acknowledgments}

The work of the Russian co-authors was partially supported by RFBR, research project No. 13-02-12175 ofi_m.

\section{References}

[1] J. Linsley, Evidence for a primary cosmic-ray particle with energy $10^{20} \mathrm{eV}$, Phys. Rev. Lett. 10 (Feb, 1963) $146-148$.

[2] K. Greisen, End to the cosmic-ray spectrum?, Phys. Rev. Lett. 16 (Apr, 1966) 748-750.

[3] G. T. Zatsepin and V. A. Kuz'min, Upper Limit of the Spectrum of Cosmic Rays, Soviet J. of Experimental and Theoretical Physics Letters 4 (Aug., 1966) 78. 
[4] A. Aab et al., Searches for anisotropies in the arrival directions of the highest energy cosmic rays detected by the Pierre Auger Observatory, The Astrophysical Journal 804 (2015), no. 1 15, [arXiv:1411.6111].

[5] R. Benson and J. Linsley, Satellite observation of cosmic ray air showers, in Proc. 17th Int. Cosmic Ray Conf. (Paris), vol. 8, pp. 145-148, 1981.

[6] D. Bugrov et al., Modeling of space telescopes for observation of tracks produced in the Earth's atmosphere by extremely high energy cosmic rays, in Mathematical modeling of complex information processing systems (V. Sadovnichii and E. Doger Guerero, eds.), pp. 98-118, Lomonosov Moscow State University Press, 2001.

[7] M. Pallavicini, R. Pesce, A. Petrolini, and A. Thea, The observation of extensive air showers from an Earth-orbiting satellite, Astropart. Phys. 35 (Feb., 2012) 402-420.

[8] L. Scarsi et al., AIR WATCH: a space mission to observe the UV fluorescence induced in the Earth atmosphere by extreme-energy cosmic radiation, in EUV, X-Ray, and Gamma-Ray Instrumentation for Astronomy IX (O. H. Siegmund and M. A. Gummin, eds.), vol. 3445 of SPIE Conference Series, pp. 505-515, Nov., 1998.

[9] F. W. Stecker et al., Observing the Ultrahigh Energy Universe with OWL Eyes, Nuclear Physics B Proceedings Supplements 136 (Nov., 2004) 433-438, [astro-ph/ 0408162 ].

[10] J. F. Krizmanic, J. W. Mitchell, and R. E. Streitmatter, Optimization of the Orbiting Wide-angle Light Collectors (OWL) mission for charged-particle and neutrino astronomy, in Proc. 32nd Int. Cosmic Ray Conf. (Beijing), p. 1085, July, 2013.

[11] M. Bertaina and E. Parizot, The JEM-EUSO mission: a space observatory to study the origin of Ultra-High Energy Cosmic Rays, Nucl. Phys. B Proc. Suppl. 256 (Nov., 2014) 275-286.

[12] A. Petrolini, Ultra-high energy cosmic particles studies from space: Super-EUSO, a possible next-generation experiment, Nucl. Inst. Methods in Phys. Res. A 630 (Feb., 2011) 131-135, [arXiv:0909.5220].

[13] M. Panasyuk et al., Detector of Extreme Energy Cosmic Rays on Board Lomonosov Satellite, J. of Cosmology 18 (2012) 7964-7977.

[14] B. A. Khrenov et al., Pioneering space based detector for study of cosmic rays beyond GZK limit, in European Physical J. Web of Conf., vol. 53, p. 9006, June, 2013.

[15] M. I. Panasyuk et al., The current status of orbital experiments for UHECR studies, arXiv:1501.0636.

[16] A. Haungs and JEM-EUSO Collaboration, Physics goals and status of JEM-EUSO and its test experiments, ArXiv e-prints (Apr., 2015) [arXiv: 1504.0259$].$

[17] S. Sharakin et al., Development of optical systems for the KLYPVE experiment, in Proc. 34th Int. Cosmic Ray Conf. (The Hague), 2015. ID 1171.

[18] Y. Kawasaki et al., The focal surface of the JEM-EUSO instrument, Astrophysics and Space Sciences Transactions 7 (2011), no. 2 167-169.

[19] M. D. Rodríguez Frías et al., The Atmospheric Monitoring System of the JEM-EUSO space mission, in European Physical Journal Web of Conferences, vol. 89 of European Physical Journal Web of Conferences, p. 2007, Mar., 2015. arXiv:1501.0482. 